\title{
Some applications of the Hermit-Hadamard inequality for log-convex functions in quantum divergence
}

\author{
Fatemeh Hassanzad ${ }^{1}$, Hossien Mehri-Dehnavi ${ }^{1}$, and Hamzeh Agahi ${ }^{2}$ \\ ${ }^{1}$ Babol Noshirvani University of Technology \\ ${ }^{2}$ Baol Noshirvani University of Technology
}

July 23, 2021

\begin{abstract}
One of the beautiful and very simple inequalities for a convex function is the Hermit-Hadamard inequality [S. Mehmood, et. al. Math. Methods Appl. Sci., 44 (2021) 3746], [S. Dragomir, et. al., Math. Methods Appl. Sci., in press]. The concept of log-convexity is a stronger property of convexity. Recently, the refined Hermit-Hadamard's inequalities for log-convex functions were introduced by researchers [C. P. Niculescu, Nonlinear Anal. Theor., 75 (2012) 662]. In this paper, by the Hermit-Hadamard inequality, we introduce two parametric Tsallis quantum relative entropy, two parametric Tsallis-Lin quantum relative entropy and two parametric quantum Jensen-Shannon divergence in quantum information theory. Then some properties of quantum Tsallis-Jensen-Shannon divergence for two density matrices are investigated by this inequality. \newline \textbf\{Keywords: $\}$ $\backslash$ textit\{ Hermit-Hadamard's inequality; log-convexity; Density matrices; Quantum relative entropy; Tsallis quantum relative entropy; quantum Jensen-Shannon divergence divergence.
\end{abstract}

\section{Hosted file}

Hasanzadv7.pdf available at https://authorea.com/users/427238/articles/531450-someapplications-of-the-hermit-hadamard-inequality-for-log-convex-functions-in-quantumdivergence 